\title{
The strange case of SN 2011ja and its host
}

\section{Sayan Chakraborti ${ }^{1}$, Alak Ray ${ }^{2}$, Randall Smith ${ }^{1}$, Stuart Ryder ${ }^{3}$, Naveen Yadav ${ }^{2}$, Firoza Sutaria ${ }^{4}$, Vikram V. Dwarkadas ${ }^{5}$, Poonam Chandra $^{6}$, David Pooley ${ }^{7}$, and Rupak Roy ${ }^{8}$}

\author{
${ }^{1}$ Harvard Smithsonian Center for Astrophysics, 60 Garden Street, Cambridge MA 02138, USA \\ email: schakraborti@fas.harvard.edu \\ ${ }^{2}$ Tata Institute of Fundamental Research, Homi Bhabha Road, Colaba, Mumbai 400005, India \\ ${ }^{3}$ Australian Astronomical Observatory, P.O. Box 915, North Ryde, NSW 1670, Australia \\ ${ }^{4}$ Indian Institute of Astrophysics, Koramangala, Bangalore, India \\ ${ }^{5}$ Department of A\&A, University of Chicago, 5640 S Ellis Avenue, Chicago, IL 60637, USA \\ ${ }^{6}$ Department of Physics, Royal Military College of Canada, Kingston, ON, K7K 7B4, Canada \\ ${ }^{7}$ Department of Physics, Sam Houston State University, Huntsville, TX, USA \\ ${ }^{8}$ Aryabhatta Research Institute of Observational Sciences, Manora peak, Nainital, India
}

\begin{abstract}
SN 2001ja was observed twice in three months using the Chandra X-Ray Observatory. The X-ray flux could be due to interaction with the circumstellar medium, perhaps dominated by the reverse shock heated thermal plasma, or from inverse Compton scattering at the forward shock. In both cases, for a steady wind-like circumstellar density profile, the X-ray flux is expected to fall off as a power law or faster. But the flux from the position of SN 2011ja, increased by a factor of three between these observations. In this presentation, we investigated possible reasons, including contamination from other astrophysical sources such as a X-Ray Binary, within the Chandra's resolution, in the host galaxy using our observations, modelling and pre-explosion Chandra/XMM data.
\end{abstract}

Keywords. Stars: Mass Loss - Supernovae: Individual: SN 2011ja — shock waves — circumstellar matter — radio continuum: general — X-rays: general

\section{Introduction}

Type IIP supernovae have prominent P Cygni features of hydrogen at peak luminosity. Doggett and Branch (1985) show that their optical light curves have plateaus of $\sim 100$ days. The plateau arises as their progenitors retain extended hydrogen envelopes until the time of core collapse. Popov (1993) found that the duration of the plateau phase has a strong dependence on the mass of the hydrogen envelope. These arguments and direct pre-explosion imaging by Smartt et al. (2009) suggest that these stars exploded as red supergiants. Smith et al. (2011) found that half of the core collapse supernovae in their sample are type IIP. Red supergiants are found in the Local Group with masses up to $25 M_{\odot}$, but Smartt et al. (2009) did not find any with masses greater than $17 M_{\odot}$ as progenitors of type IIP supernovae. Many solutions have been suggested for this red supergiant problem. O'Connor and Ott (2011) suggested that the ZAMS mass, metallicity, rotation and mass-loss prescription controls the compactness of the stellar core at bounce which determines whether a core-collapse supernova will fail. Walmswell and Eldridge (2012) have suggested circumstellar dust as a solution to the problem of the missing massive progenitors. In this situation, understanding the nature, amount and variability of mass loss from the progenitors of type IIP supernovae is crucial for resolving this puzzle. 


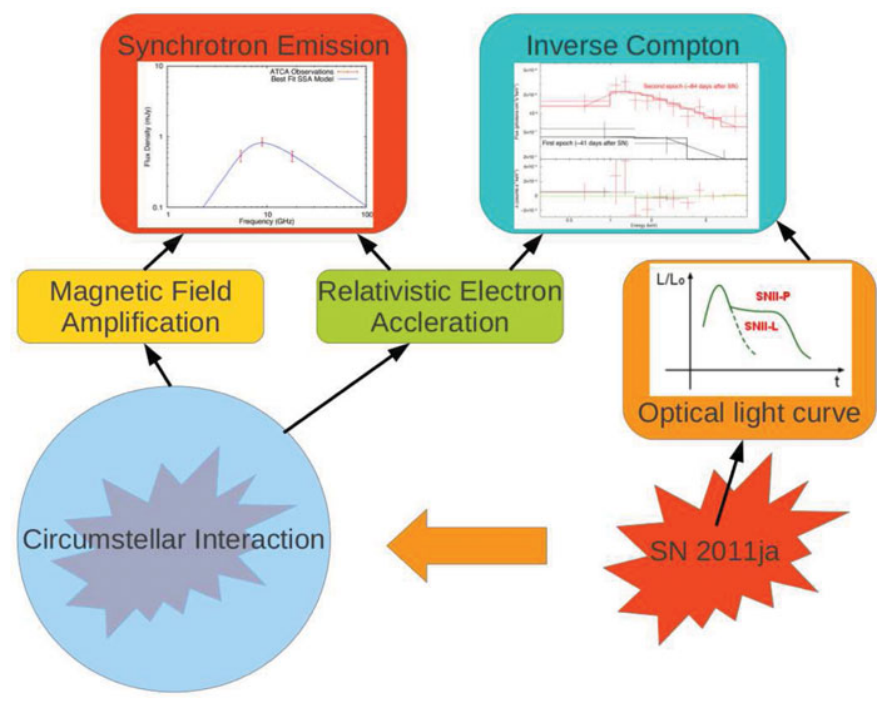

Figure 1. Radio synchrotron and X-ray inverse Compton emission from SN 2011ja.

\section{Results}

Supernova ejecta shocks circumstellar matter set up by stellar wind. Since the ejecta $\left(\sim 10^{4} \mathrm{~km} \mathrm{~s}^{-1}\right)$ moves about a thousand times faster than the stellar wind $(\sim 10 \mathrm{~km}$ $\mathrm{s}^{-1}$ ), it probes a millennium of red supergiant mass loss history in a year. This interaction accelerates particles to relativistic energies, which then lose energy via synchrotron radiation in the shock-amplified magnetic fields and inverse Compton scattering against optical photons from the supernova (Fig 1). Chevalier et al. (2006) have shown that these processes produce separate signatures in the radio and X-rays. Chakraborti et al. (2012) have demonstrated that combining radio and X-ray spectra allows one to break the degeneracy between the efficiencies of shock acceleration and field amplification. Chakraborti et al. (2013) use X-rays observations of SN 2011ja from Chandra and radio observations from the Australia Telescope Compact Array (ATCA) to study the relative importance of particle acceleration and magnetic field amplification for producing the non thermal radiation from SN 2011ja. Chakraborti et al. (2013) use a multiple Chandra observation of SN 2011ja to establish variable mass loss from the progenitor.

\section{References}

J. B. Doggett \& D. Branch, AJ 90, 2303-2311 (1985).

D. V. Popov, ApJ 414, 712-716 (1993).

S. J. Smartt, J. J. Eldridge, R. M. Crockett \& J. R. Maund, MNRAS 395, 1409-1437 (2009), 0809.0403.

N. Smith, W. Li, A. V. Filippenko \& R. Chornock, MNRAS 412, 1522-1538 (2011), 1006. 3899.

E. O'Connor \& C. D. Ott, ApJ 730, 70 (2011), 1010.5550.

J. J. Walmswell \& J. J. Eldridge, MNRAS 419, 2054-2062 (2012), 1109.4637.

R. A. Chevalier, C. Fransson \& T. K. Nymark, ApJ 641, 1029-1038 (2006), arXiv:astro$\mathrm{ph} / 0509468$.

S. Chakraborti, N. Yadav, A. Ray, R. Smith, P. Chandra, \& D. Pooley, ApJ 761, 100 (2012), 1206.4033.

S. Chakraborti, A. Ray, R. Smith, S. Ryder, N. Yadav, F. Sutaria, V. V. Dwarkadas, P. Chandra, D. Pooley, \& R. Roy, ArXiv e-prints (2013), 1302.7067. 